\title{
Phytoprotection
}

\section{Caractérisation pathologique et physiologique de deux populations de Verticillium albo-atrum isolées de la luzerne et de la pomme de terre}

\author{
T. Barasubiye, C. Richard et D. Dostaler
}

Volume 75, numéro 2, 1994

URI : https://id.erudit.org/iderudit/706052ar

DOI : https://doi.org/10.7202/706052ar

Aller au sommaire du numéro

Éditeur(s)

Société de protection des plantes du Québec (SPPQ)l

ISSN

0031-9511 (imprimé)

1710-1603 (numérique)

Découvrir la revue

Citer cet article

Barasubiye, T., Richard, C. \& Dostaler, D. (1994). Caractérisation pathologique et physiologique de deux populations de Verticillium albo-atrum isolées de la luzerne et de la pomme de terre. Phytoprotection, 75(2), 53-67.

https://doi.org/10.7202/706052ar
Résumé de l'article

Le pouvoir pathogène et la croissance de souches de Verticillium alboatrum isolées de la luzerne (Medicago sativa) et de la pomme de terre (Solarium tuberosum) ont été déterminés. D'une part, sur la luzerne, les souches de la luzerne étaient plus virulentes que celles de la pomme de terre. D'autre part, seules les souches de la pomme de terre ont attaqué la pomme de terre. Sur la gélose dextrosée à la pomme de terre, la croissance optimale a été obtenue à $20^{\circ} \mathrm{C}$ pour les souches de la pomme de terre, et à $20-25^{\circ} \mathrm{C}$ pour les souches de la luzerne. La température de $30^{\circ} \mathrm{C}$ n'a pas été létale pour les souches de la luzerne, alors que $75 \%$ des souches de la pomme de terre ont été tuées à cette température. Avec le milieu de Fries, la masse sèche du mycélium des souches de la pomme de terre était de trois à quatre fois supérieure à celle des souches de la luzerne. À l'opposé, un milieu contenant du chlorate de potassium a inhibé d'une façon significative la croissance des souches de la pomme de terre comparativement aux souches de la luzerne. Ces résultats indiquent que les souches de la luzerne et celles de la pomme de terre constituent deux groupes pathologiquement et physiologiquement distincts. 


\title{
Caractérisation pathologique et physiologique de deux populations de Verticillium albo-atrum isolées de la luzerne et de la pomme de terre
}

\author{
Tharcisse Barasubiye ${ }^{1}$, Claude Richard ${ }^{2}$ et Daniel Dostaler ${ }^{1}$
}

Reçu 1994-04-05; accepté 1994-10-25

Le pouvoir pathogène et la croissance de souches de Verticillium alboatrum isolées de la luzerne (Medicago sativa) et de la pomme de terre (Solanum tuberosum) ont été déterminés. D'une part, sur la luzerne, les souches de la luzerne étaient plus virulentes que celles de la pomme de terre. D'autre part, seules les souches de la pomme de terre ont attaqué la pomme de terre. Sur la gélose dextrosée à la pomme de terre, la croissance optimale a été obtenue à $20^{\circ} \mathrm{C}$ pour les souches de la pomme de terre, et à $20-25^{\circ} \mathrm{C}$ pour les souches de la luzerne. La température de $30^{\circ} \mathrm{C}$ n'a pas été létale pour les souches de la luzerne, alors que $75 \%$ des souches de la pomme de terre ont été tuées à cette température. Avec le milieu de Fries, la masse sèche du mycélium des souches de la pomme de terre était de trois à quatre fois supérieure à celle des souches de la luzerne. À l'opposé, un milieu contenant du chlorate de potassium a inhibé d'une façon significative la croissance des souches de la pomme de terre comparativement aux souches de la luzerne. Ces résultats indiquent que les souches de la luzerne et celles de la pomme de terre constituent deux groupes pathologiquement et physiologiquement distincts.

Barasubiye, T., C. Richard, and D. Dostaler. 1994. Pathological and physiological characterization of two Verticillium albo-atrum populations isolated from alfalfa and potato. PHYTOPROTECTION 75: 53-67.

Pathogenicity and growth of strains of Verticillium albo-atrum recovered from alfalfa (Medicago sativa) and potato (Solanum tuberosum) were determined. On alfalfa, the alfalfa strains were more virulent than the potato strains, and only the potato strains were virulent on potato. The optimum temperature for growth was $20^{\circ} \mathrm{C}$ for potato strains, and $20-25^{\circ} \mathrm{C}$ for alfalfa strains on potato dextrose agar. At $30^{\circ} \mathrm{C}$, no alfalfa strain was killed whereas this temperature was lethal for $75 \%$ of the potato strains. On Fries medium, the potato strains produced three to four times more dry weight than the alfalfa strains. However, on a solid medium amended with potassium chlorate, a significant inhibition was noted for potato strains as compared to alfalfa strains. These results indicate that $V$. albo-atrum strains isolated from alfalfa and potato form two pathologically and physiologically distinct groups.

1. Département de phytologie, Faculté des sciences de l'agriculture et de l'alimentation, Université Laval, Québec (Québec), Canada G1K 7P4

2. Centre de recherche, Agriculture et Agro-alimentaire Canada, 2560, boul. Hochelaga, Sainte-Foy (Québec), Canada G1V 2J3. Auteur correspondant. Contribution ${ }^{\circ} 499$ 


\section{INTRODUCTION}

Le Verticillium albo-atrum Reinke \& Berthier ( $\mathrm{Vaa}$ ) est un agent pathogène de la verticilliose de la luzerne (Medicago sativa L.) et de la pomme de terre (Solanum tuberosum L.). Depuis 1918, cette maladie destructrice entrave la production de luzerne dans les pays nordiques d'Europe (Kreitlow 1962). En 1925, ce champignon pathogène a été isolé de la pomme de terre en Ontario (Berkeley et al. 1931). Par ailleurs, le champignon entraîne des pertes dans les productions de pomme de terre de I'Île-du-Prince-Édouard depuis 1930 (Ayers et Hurst 1939).

Du point de vue climatique, le $V$ aa est adapté au climat tempéré humide ( $\mathrm{Ga}$ gné et Richard 1982). Dans les régions à climat chaud, c'est le V. dahliae Kleb. qui est l'agent pathogène principal de la verticilliose, notamment chez la pomme de terre (Nachmias et Krikun 1985). Au Canada, la verticilliose de la pomme de terre est attribuée au Vaa (McKeen et Thorpe 1981; Platt et Sanderson 1987). Platt (1986) rapporte des pertes de rendement de $40 \%$ chez les cultivars de pomme de terre sensibles à la verticilliose.

La verticilliose est relativement récente chez la luzerne dans les États américains (Gagné et Richard 1982). Elle a été signalée au Québec pour la première fois en 1962, par Aubé et Sackston (1964), dans des parcelles expérimentales de luzerne. Toutefois, la maladie a été éradiquée. Plus tard, en 1976, la verticilliose est apparue avec une grande intensité, principalement dans les États américains de Washington et d'Oregon (Graham et al. 1977). Par la suite, la maladie s'est propagée aussi bien dans les États américains que dans les provinces canadiennes (Gordon et al. 1989; Gossen et Jesperson 1990; Martin et Boswall 1989; Nicholls et al. 1987).

D'une part, les souches de Vaa semblent avoir une spécificité pathologique à l'égard de leur plante-hôte autant en Europe qu'en Amérique du Nord (Correll et al. 1988; Heale et Isaac 1963). $D^{\prime}$ autre part, les études sont contradic- toires quant à la virulence des souches selon leur origine géographique. Ainsi, Christen et al. (1983) n'ont pas trouvé de différence significative de virulence entre des souches américaines de $\mathrm{Vaa}$ isolées de la luzerne et des souches européennes. Par contre, les travaux antérieurs de Flood et al. (1978) montraient que des souches nord-américaines du Vaa étaient plus virulentes chez la luzerne que des souches européennes.

Par ailleurs, Isaac et Lloyd (1959) ont obtenu un niveau d'infection de $88 \%$ chez la pomme de terre inoculée avec des souches de Vaa isolées de la luzerne. À l'opposé, dans une expérience semblable, Christen et French (1982) n'ont pas observé d'infection chez la pomme de terre inoculée avec des souches de Vaa obtenues de la luzerne. À la lumière de ces derniers travaux et en considérant les dates différentes des premiers relevés, voire de l'introduction des verticillioses de la luzerne et de la pomme de terre en Amérique du Nord, les souches de l'agent pathogène qui provoquent la verticilliose chez la pomme de terre seraient différentes des souches retrouvées chez la luzerne.

Au moyen d'inoculations chez la luzerne et la pomme de terre en milieu contrôlé ainsi que de différentes mesures de croissance in vitro, la présente étude vise à préciser la spécificité pathologique et les caractéristiques physiologiques des souches nordaméricaines du Vaa.

\section{MATÉRIEL ET MÉTHODES}

\section{Souches de Vaa}

Trente-cinq souches de Vaa ont été utilisées. Ces souches, principalement isolées en Amérique du Nord, comprenaient 20 souches obtenues de la luzerne et 15 obtenues de la pomme de terre (tableau 1). Les souches monoconidiennes ont été conservées à $4^{\circ} \mathrm{C}$ sur une gélose dextrosée à la pomme de terre (PDA, Difco) recouverte d'une huile minérale stérile. Les cultures actives obtenues en repiquant le mycélium sur PDA en boîtes de Pétri étaient maintenues à l'obscurité, à $22^{\circ} \mathrm{C}$, pendant $10 \mathrm{j}$. 
Tableau 1. Souches de Verticillium albo-atrum isolées de la luzerne et de la pomme de terre

\begin{tabular}{|c|c|c|c|}
\hline Souche & Hôte & Origine & Fournisseur \\
\hline \multicolumn{4}{|l|}{ Série 1} \\
\hline $424^{\S}$ & Medicago sativa & Alberta & H.C. Huang \\
\hline 426 & Medicago sativa & Alberta & S.F. Hwang $(R-9)^{\dagger}$ \\
\hline 434 & Medicago sativa & Ontario & P.K. Basu (\#3) \\
\hline 224 & Medicago sativa & Maryland & J.H. Graham (w10) \\
\hline 380 & Medicago sativa & Québec & C. Richard \\
\hline 403 & Medicago sativa & Québec & C. Richard \\
\hline 451 & Medicago sativa & New York & $\begin{array}{l}\text { G.C. Bergostrom } \\
\text { (vaa } 007 \text { NY 83) }\end{array}$ \\
\hline 227 & Medicago sativa & France & J. Gondran \\
\hline 428 & Medicago sativa & Californie & R.A. Khan (va-25) \\
\hline 423 & Medicago sativa & Washington & R.N. Peaden (wa 40-3) \\
\hline 437 & Solanum tuberosum & Île-du-Prince-Édouard & M. Celetti (vaa-1401) \\
\hline 170 & Solanum tuberosum & Québec & C. Richard \\
\hline 470 & Solanum tuberosum & Québec & T. Barasubiye \\
\hline 471 & Solanum tuberosum & Québec & T. Barasubiye \\
\hline 472 & Solanum tuberosum & Québec & T. Barasubiye \\
\hline 473 & Solanum tuberosum & Québec & T. Barasubiye \\
\hline 442 & Solanum tuberosum & Maine & R.A. Henn (2c-3) \\
\hline \multicolumn{4}{|l|}{ Série 2} \\
\hline 435 & Medicago sativa & Ontario & P.K. Basu (\#4) \\
\hline 410 & Medicago sativa & Québec & C. Richard \\
\hline 433 & Medicago sativa & Saskatchewan & P.K. Basu (\#2) \\
\hline 228 & Medicago sativa & France & J. Gondran \\
\hline 443 & Medicago sativa & Californie & P. Grybauskas (MD\#171) \\
\hline 448 & Medicago sativa & Californie & T.R. Gordon (CA4) \\
\hline 447 & Medicago sativa & Colombie-Britannique & K. Leath (Laws 82-055) \\
\hline 450 & Medicago sativa & New York & $\begin{array}{l}\text { G.C. Bergostrom } \\
\text { (vaa } 004 \text { NY 83) }\end{array}$ \\
\hline 445 & Medicago sativa & Pennsylvanie & K. Leath (vp-11) \\
\hline 282 & Medicago sativa & Washington & J. Sheppard (95) \\
\hline 479 & Solanum tuberosum & Québec & T. Barasubiye \\
\hline 480 & Solanum tuberosum & Québec & T. Barasubiye \\
\hline 481 & Solanum tuberosum & Québec & T. Barasubiye \\
\hline 482 & Solanum tuberosum & Québec & T. Barasubiye \\
\hline 485 & Solanum tuberosum & Québec & T. Barasubiye \\
\hline 487 & Solanum tuberosum & Québec & T. Barasubiye \\
\hline 542 & Solanum tuberosum & Cuba & Ottawa (DAOM-145760) \\
\hline 441 & Solanum tuberosum & Maine & R.A. Henn (3D-3B) \\
\hline
\end{tabular}

$\S$ Numéro de collection des souches de V. albo-atrum au Centre de recherche de Sainte-Foy, Agriculture et Agro-alimentaire Canada.

† Les numéros originaux des souches sont indiqués entre parenthèses. 


\section{Luzerne}

Deux cultivars de luzerne, Oneida VR et Saranac, respectivement résistant et sensible à la verticilliose, ont été inoculés avec les souches de Vaa (tableau 1) en cabinet de croissance. Chaque cultivar a été semé individuellement dans des caissettes de $26 \mathrm{~cm} \times 51 \mathrm{~cm} \times 6 \mathrm{~cm}$ contenant un terreau pasteurisé à la vapeur. Les caissettes ont été placées dans une chambre de croissance à $19^{\circ} \mathrm{C}$ le jour et $16^{\circ} \mathrm{C}$ la nuit, avec un éclairage

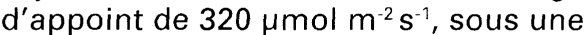
photopériode de $16 \mathrm{~h}$. Sept jours après le semis, nous avons inoculé les plantes avec la souche A2 du Rhizobium meliloti Dangeard.

\section{Pomme de terre}

En serre, nous avons estimé le pouvoir pathogène des mêmes souches de $V a a$ avec deux cultivars de pomme de terre, Atlantic et Superior, respectivement tolérant et sensible à la verticilliose. Des tubercules de classe Élite $1 \mathrm{du} \mathrm{cv}$. Superior et de classe Fondation du cv. Atlantic ont été plantés dans des pots $(15 \mathrm{~cm}$ diam) contenant un substrat composé de mousse de tourbe, de terre noire, de perlite, de vermiculite et de sable (38:28:14:12:8, v:v:v:v:v). Ce mélange a été préalablement stérilisé à la vapeur. Après la levée, les plantules de pomme de terre ont été arrosées au besoin sans être fertilisées. En hiver, la pomme de terre a été maintenue à $22^{\circ} \mathrm{C}$ le jour et $18^{\circ} \mathrm{C}$ la nuit, avec un éclairage de $220 \mu \mathrm{mol} \mathrm{m} \mathrm{m}^{-2} \mathrm{~s}^{-1}$, sous une photopériode de $14 \mathrm{~h}$. En été, les conditions moyennes de croissance étaient de $27^{\circ} \mathrm{C}$, sous un éclairage naturel plus un éclairage d'appoint de $170 \mu \mathrm{mol}$ $\mathrm{m}^{-2} \mathrm{~s}^{-1}$ et sous une photopériode de $14 \mathrm{~h}$.

\section{Préparation de l'inoculum}

Les conidies de Vaa ont été décrochées avec $5 \mathrm{~mL}$ d'eau distillée stérile à partir de cultures individuelles âgées de $10 \mathrm{j}$. Des fioles Erlenmeyer de $250 \mathrm{~mL}$ contenant $100 \mathrm{~mL}$ de milieu liquide Czapek (Difco) ont été ensemencées avec $3 \mathrm{~mL}$ de suspension de conidies. Après l'ensemencement, les cultures ont été placées sur un agitateur à $150 \mathrm{r} \mathrm{min}^{-1}$ pendant $3 \mathrm{j}$ à l'obscurité, à $22^{\circ} \mathrm{C}$. La concentration de l'inoculum a été ajustée à $2 \times 10^{6}$ conidies $\mathrm{mL}^{-1}$.

\section{Inoculation, traitement de la} luzerne et prise de données

Les racines de la luzerne âgée de $50 \mathrm{j}$ ont été lavées à l'eau du robinet. Les racines et les tiges ont été coupées à $5 \mathrm{~cm}$ du collet, puis les plantes ont été trempées dans l'inoculum individuel des souches de Vaa série 1 (tableau 1) pendant 30 min (Graham et al. 1977). Les plantes inoculées ont été transplantées dans des pots $(10 \mathrm{~cm}$ diam) contenant le substrat Pro-Mix BX (Les Tourbières Premier Ltée, Québec) stérilisé à la vapeur. Par la suite, nous avons placé les plantes dans deux cabinets de croissance réglés à $22^{\circ} \mathrm{C}$ le jour et $18^{\circ} \mathrm{C}$ la nuit, sous un éclairage de $320 \mu \mathrm{mol}$ $\mathrm{m}^{-2} \mathrm{~s}^{-1}$ et une photopériode de $16 \mathrm{~h}$. Nous avons utilisé un dispositif en parcelles partagées (Steel et Torrie 1980) avec cinq répétitions. Les cultivars Oneida VR et Saranac ont été placés en parcelles principales, avec les souches de Vaa série 1 et les plantes non inoculées (témoins) en sous-parcelles. Un pot contenant cinq plantes constituait l'unité expérimentale. Nous avons arrosé les plantes au besoin, sans apport subséquent d'éléments fertilisants.

Chez la luzerne, l'intensité de la verticilliose a été évaluée $21 \mathrm{j}$ après l'inoculation. Le flétrissement des feuilles ( $1=$ plante saine et $5=$ plante morte) et le brunissement des racines $(1=$ racine saine et $5=$ racine morte) ont été notés selon les échelles de Graham et al. (1977). Les repousses des plantes ont été coupées à $5 \mathrm{~cm}$ au-dessus du collet, séchées à $70^{\circ} \mathrm{C}$ pendant $48 \mathrm{~h}$, et pesées pour évaluer le rendement de la biomasse aérienne. Une expérience semblable a été effectuée avec la série 2 des souches de Vaa (tableau 1).

\section{Inoculation, traitement de la pomme de terre et prise de données}

Les racines de pomme de terre (cv. Atlantic et cv. Superior) âgées de $30 \mathrm{j}$ ont été lavées à l'eau du robinet et le tubercule de semence a été enlevé. Les semenceaux ont ensuite été trempés pendant $30 \mathrm{~min}$ dans l'inoculum des souches de Vaa série 1 (tableau 1) ajusté à $2 \times 10^{6}$ conidies $\mathrm{mL}^{-1}$. Les plantes ont été gardées $7 \mathrm{j}$ à $18^{\circ} \mathrm{C}$ sous un éclairage de $80 \mu \mathrm{mol} \mathrm{m}^{-2} \mathrm{~s}^{-1}$ et une photopé- 
riode de $16 \mathrm{~h}$. Après ce temps d'incubation, les plantes ont été transférées en serre selon un dispositif en parcelles partagées avec trois répétitions. Les deux cultivars ont été placés en parcelles principales, avec les souches de $\mathrm{Vaa}$ et les témoins en sous-parcelles. Un pot contenant deux plantes constituait I'unité expérimentale. Dans cette expérience réalisée en été, les plantes ont poussé à la température moyenne de $27^{\circ} \mathrm{C}$ sous un éclairage naturel plus un éclairage d'appoint de $170 \mu \mathrm{mol} \mathrm{m} \mathrm{m}^{-2} \mathrm{~s}^{-1}$ avec une photopériode de $14 \mathrm{~h}$. Nous avons appliqué une fertilisation hebdomadaire avec un engrais soluble 15-2020 (N-P-K).

À l'aide de l'échelle réduite de Corsini et al. (1985) $(1=$ plante saine et $5=$ $>90 \%$ des feuilles flétries), nous avons estimé l'intensité de la verticilliose de la pomme de terre $35 \mathrm{j}$ après l'inoculation et mesuré le rendement en tubercules. Nous avons effectué une deuxième expérience en serre avec les souches de Vaa série 2 (tableau 1) en hiver, à $22^{\circ} \mathrm{C}$ le jour et $18^{\circ} \mathrm{C}$ la nuit, sous un éclairage de $220 \mu \mathrm{mol} \mathrm{m} \mathrm{m}^{-2} \mathrm{~s}^{-1}$ et une photopériode de $14 \mathrm{~h}$.

\section{Effet de la température sur la croissance des souches de Vaa}

Des rondelles de $5 \mathrm{~mm}$ diam ont été prélevées à la marge des cultures actives des souches de Vaa pour ensemencer le centre de boîtes de Pétri de $9 \mathrm{~cm}$ diam contenant $18 \mathrm{~mL}$ de PDA. Par la suite, les boîtes de Pétri ensemencées ont été placées à l'obscurité dans des incubateurs ajustés à $5,10,15,20,22$, 25,27 et $30^{\circ} \mathrm{C}$. Un dispositif complètement aléatoire a été utilisé et l'unité expérimentale était une boîte de Pétri. Deux rayons perpendiculaires de chaque culture ont été mesurés après $10 \mathrm{j}$ de croissance. Les souches incubées à $30^{\circ} \mathrm{C}$ ont été ensemencées de nouveau sur du PDA fraîchement préparé. Après $12 \mathrm{j}$ d'incubation à $22^{\circ} \mathrm{C}$, la croissance mycélienne radiale a été notée sur la base de deux rayons perpendiculaires. Cette expérience a été répétée trois fois.

\section{Détermination de la croissance des souches de Vaa sur le milieu de Fries}

Des bouteilles de Roux de $900 \mathrm{~mL}$ contenant $120 \mathrm{~mL}$ de milieu de Fries (Dhin- gra et Sinclair 1985) ont été ensemencées avec une suspension d'inoculum de chaque souche de Vaa ajustée à une concentration de $10^{6}$ conidies $\mathrm{mL}^{-1}$. Nous avons placé les bouteilles à l'obscurité à $22^{\circ} \mathrm{C}$ selon un dispositif en blocs complets avec une bouteille comme unité expérimentale. Après $14 \mathrm{j}$ de croissance, le mycélium a été recueilli par filtration à travers une double épaisseur de mousseline de coton, puis congelé dans de l'azote liquide et lyophilisé. La masse sèche du mycélium a été mesurée et l'expérience répétée trois fois.

\section{Effet du chlorate de potassium sur la croissance radiale des souches de Vaa}

Pour évaluer la toxicité du chlorate de potassium sur les souches de Vaa, nous avons incorporé $15 \%$ de ce produit dans le milieu de Puhalla (1985). Les souches de Vaa ont été ensemencées au centre de boîtes de Pétri contenant $18 \mathrm{~mL}$ de ce milieu. Nous avons utilisé un dispositif en blocs complets avec une boîte de Pétri comme unité expérimentale. L'ensemencement sur le milieu sans chlorate de potassium constituait le traitement-témoin. Après $10 \mathrm{j} \mathrm{d}^{\prime}$ incubation à l'obscurité à $22^{\circ} \mathrm{C}$, deux rayons perpendiculaires ont été mesurés pour chaque culture. L'expérience a été répétée trois fois.

\section{Analyse statistique}

La procédure GLM (SAS Institute Inc. 1990) a été utilisée pour l'analyse de la variance. Avec les données du pouvoir pathogène obtenues en cabinet de croissance et en serre, l'interaction souches $x$ cultivars a été décomposée et nous avons calculé le rapport de variance souches intra-cultivar. Les moyennes individuelles des souches de Vaa et du traitement-témoin non inoculé ont été comparées à l'aide du test LSD protégé (Steel et Torrie 1980), tandis que les moyennes des souches de $\mathrm{Vaa}$ isolées de la luzerne et de la pomme de terre ont été comparées entre elles par des contrastes orthogonaux. Quant à la croissance sur divers milieux et à l'effet de la température, après une analyse de variance, des contrastes orthogonaux ont été utilisés pour comparer les souches de Vaa isolées de la luzerne à celles obtenues de la pomme de terre. 


\section{RÉSULTATS}

\section{Pouvoir pathogène des souches de Vaa chez la luzerne}

Les résultats de la détermination du pouvoir pathogène des souches de $V$. albo-atrum de la luzerne (Vaa-L) et de la pomme de terre (Vaa-P) série 1 sont présentés au tableau 2. Les plantes de luzerne cv. Saranac inoculées avec les souches Vaa-L 224, 380, 403, 423, 424, 426,434 et 451 ont eu des indices de flétrissement des feuilles significativement supérieurs $(P \leq 0,05)$ à ceux obtenus chez les témoins et chez les plantes inoculées avec les souches Vaa-P 437, 442, 470, 471, 472 et 473 . Enfin, les indices de flétrissement des feuilles obtenus chez les plantes inoculées avec les souches Vaa- $L(x=3,43)$ et Vaa-P $(x$ $=2,22$ ) étaient significativement différents $(P \leq 0,01)$.

Les plantes-témoins ont eu un indice de brunissement des racines significativement inférieur $(P \leq 0,05)$ à l'ensemble des indices obtenus chez les plantes inoculées avec les souches de Vaa (tableau 2). De plus, les indices de brunissement des racines obtenus chez les plantes inoculées avec les souches Vaa$L(x=3,47)$ et Vaa- $P(x=2,91)$ étaient significativement différents $(P \leq 0,01)$.

La luzerne inoculée avec les souches Vaa-L 380,403, 423, 424, 426 et 434 a eu des biomasses sèches aériennes significativement inférieures $(P \leq 0,05)$ à celle de la luzerne-témoin. La moyenne de la biomasse sèche aérienne était presque deux fois plus élevée $(P \leq 0,01)$ chez les plantes inoculées avec les souches Vaa-P comparativement aux souches Vaa-L (tableau 2).

Chez la luzerne cv. Oneida VR, les plantes inoculées avec les souches VaaL 224, 426, 428 et 434 ont eu un flétrissement des feuilles significativement accentué par rapport aux témoins (tableau 2). Toutefois, les indices de flétrissement des feuilles obtenus chez les plantes inoculées avec les souches $V$ aa$L(x=2,10)$ et Vaa- $P(x=1,70)$ étaient significativement différents $(P \leq 0,05)$.

Toutes les plantes inoculées avec les souches Vaa-P (sauf 437) et les souches Vaa-L (sauf 451 et 424 ) ont eu un indice de brunissement des racines significativement supérieur $(P \leq 0,05)$ à celui des témoins non inoculés (tableau 2). Avec la luzerne cV. Oneida VR, le rapport de variance $(F)$ n'a pas été significatif pour la biomasse sèche aérienne.

Les résultats obtenus avec les souches de Vaa série 2 sont consignés au tableau 3. Seules les plantes du cv. Saranac inoculées avec la souche 448 n'ont pas montré un indice de flétrissement des feuilles supérieur $(P \leq 0,05)$ à celui des plantes-témoins. Par contre, les plantes inoculées avec les souches Vaa-L (sauf 433 et 448 ) ont eu des indices de flétrissement des feuilles significativement supérieurs $(P \leq 0,05)$ à ceux des plantes inoculées avec les souches Vaa-P. Au sein des souches Vaa--P, les plantes inoculées avec les souches 479 , 481 et 487 ont eu des indices de flétrissement des feuilles significativement supérieurs $(P \leq 0,05)$ à celui des plantes-témoins (tableau 3 ). Toutefois, les indices de flétrissement des feuilles obtenus chez les plantes inoculées avec les souches Vaa-L $(x=3,15)$ et Vaa-P $(x=2,16)$ étaient significativement différents $(P \leq 0,01)$.

Les racines des plantes du cv. Saranac inoculées avec les souches $V$ aa- $L$ $228,282,410,433,435,443,445,447$ et 450 ont eu des indices de brunissement significativement supérieurs $(P \leq 0,05)$ aux indices obtenus chez les plantes inoculées avec les souches $V a a-P$, à l'exception de la souche 485 (tableau 3). Toutefois, les indices de brunissement des racines obtenus chez les plantes inoculées avec les souches Vaa- $L(x=$ $3,50)$ et Vaa-P $(x=2,80)$ étaient significativement différents $(P \leq 0,01)$.

Pour les rendements, la moyenne de $530 \mathrm{mg}$ pour la biomasse sèche aérienne des plantes inoculées avec les souches Vaa-P était supérieure $(P \leq 0,01)$ aux $409 \mathrm{mg}$ des plantes inoculées avec les souches Vaa-L. Les plantes du cv. Saranac inoculées avec les souches VaaP 441, 480, 482, 485 et 542 ont eu des biomasses sèches aériennes significativement supérieures $(P \leq 0,05)$ à celles des plantes-témoins (tableau 3 ). De même pour les souches Vaa-L, les plantes inoculées avec les souches 433 
et 448 ont eu des biomasses sèches supérieures $(P \leq 0,05)$ à celles des plantes-témoins.

Les plantes du cv. Oneida VR inoculés avec les souches Vaa-L 228, 435 et 443 ont eu un indice de flétrissement des feuilles significativement supérieur $(P \leq 0,05)$ à celui des plantes-témoins (tableau 3). Toutefois, les indices de flétrissement des feuilles obtenus chez les plantes inoculées avec les souches Vaa-L $(x=2,30)$ et Vaa-P $(x=1,89)$ étaient significativement différents $(P \leq 0,01)$.

Au niveau des racines (tableau 3), les indices de brunissement de la luzerne cV. Oneida VR inoculées avec les souches Vaa-L $(x=2,83)$ et Vaa-P $(x=$ 2,62 ) étaient significativement différents $(P \leq 0,01)$, et la biomasse sèche aérienne a varié en fonction des souches.

Tableau 2. Pouvoir pathogène des souches Vaa-L et Vaa-P envers deux cultivars de luzerne (série 1)

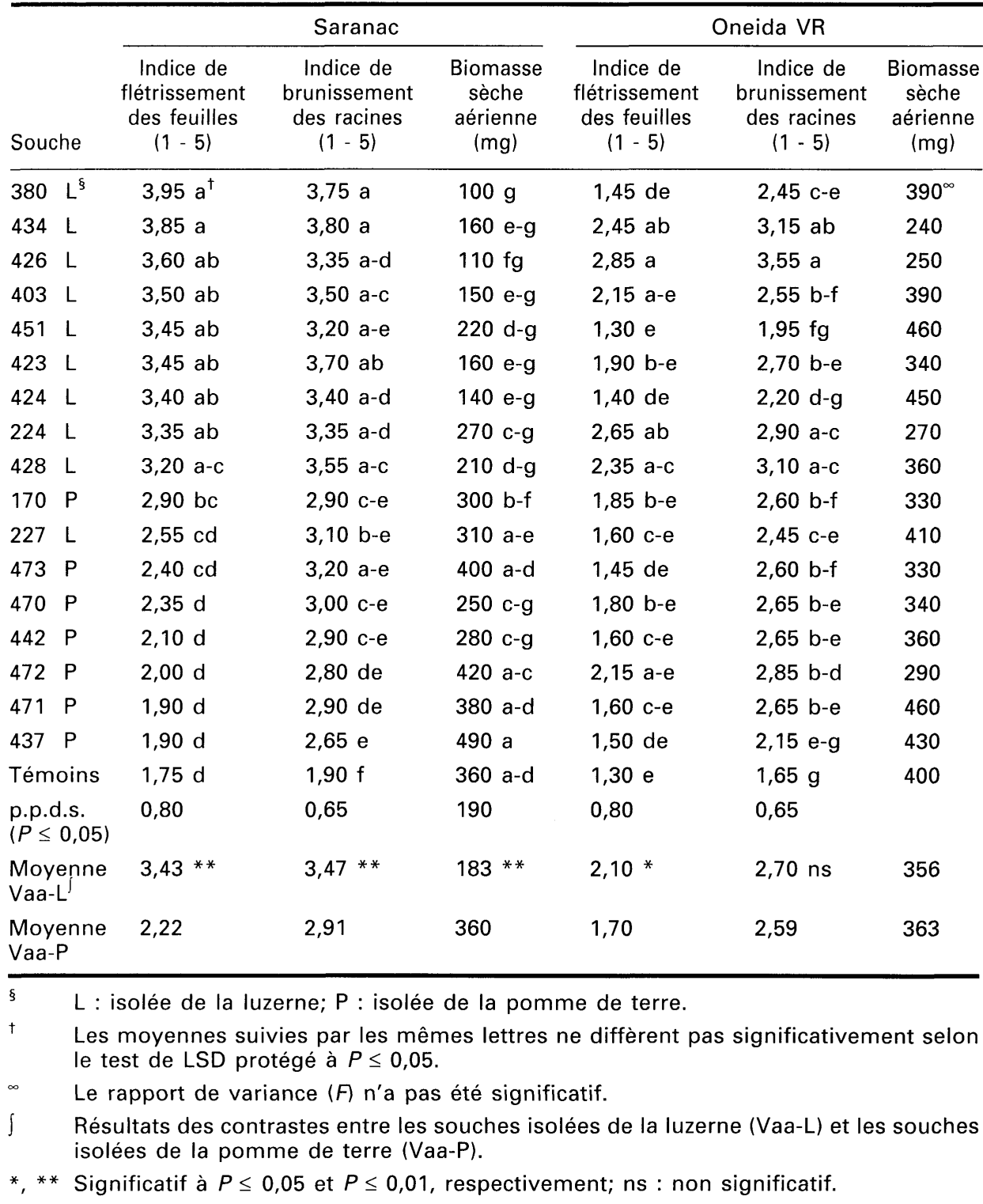


Tableau 3. Pouvoir pathogène des souches Vaa-L et Vaa-P envers deux cultivars de luzerne (série 2)

\begin{tabular}{|c|c|c|c|c|c|c|}
\hline \multirow[b]{2}{*}{ Souche } & \multicolumn{3}{|c|}{ Saranac } & \multicolumn{3}{|c|}{ Oneida VR } \\
\hline & $\begin{array}{c}\text { Indice de } \\
\text { flétrissement } \\
\text { des feuilles } \\
(1-5)\end{array}$ & $\begin{array}{c}\text { Indice de } \\
\text { brunissement } \\
\text { des racines } \\
(1-5)\end{array}$ & $\begin{array}{l}\text { Biomasse } \\
\text { sèche } \\
\text { aérienne } \\
\text { (mg) }\end{array}$ & $\begin{array}{c}\text { Indice de } \\
\text { flétrissement } \\
\text { des feuilles } \\
(1-5)\end{array}$ & $\begin{array}{c}\text { Indice de } \\
\text { brunissement } \\
\text { des racines } \\
(1-5)\end{array}$ & $\begin{array}{l}\text { Biomasse } \\
\text { sèche } \\
\text { aérienne } \\
\text { (mg) }\end{array}$ \\
\hline $443 \mathrm{~L}^{\S}$ & $3,60 \mathrm{a}^{\dagger}$ & $3,83 a$ & $260 \mathrm{~g}$ & $2,78 \mathrm{a}$ & $3,30 \mathrm{a}$ & $250 \mathrm{~h}$ \\
\hline $450 \mathrm{~L}$ & 3,58 a & $3,53 \mathrm{ab}$ & $290 \mathrm{fg}$ & $2,34 a-d$ & $2,85 b-e$ & 350 e-h \\
\hline $410 \mathrm{~L}$ & 3,45 a & $3,68 \mathrm{ab}$ & $440 \mathrm{~b}-\mathrm{f}$ & 2,38 a-c & 3,10 a-c & 540 a-d \\
\hline $282 \mathrm{~L}$ & 3,45 a & $3,48 a b$ & 350 e-g & $2,15 b-e$ & $2,58 \mathrm{~d}-\mathrm{h}$ & $300 \mathrm{gh}$ \\
\hline $435 \mathrm{~L}$ & $3,38 a b$ & 3,78 a & $420 \mathrm{c}-\mathrm{g}$ & 2,78 a & $3,21 a b$ & 580 a-c \\
\hline $228 \mathrm{~L}$ & $3,35 a b$ & $3,65 a b$ & 350 e-g & $2,48 a b$ & $2,78 \mathrm{c}-\mathrm{f}$ & $410 \mathrm{~d}-\mathrm{h}$ \\
\hline $445 \mathrm{~L}$ & $3,28 a b$ & $3,55 a b$ & 490 b-e & $2,30 b-d$ & $2,55 \mathrm{~d}-\mathrm{g}$ & 360 e-h \\
\hline $447 \quad \mathrm{~L}$ & $3,00 \mathrm{bc}$ & $3,33 \mathrm{bc}$ & $310 \mathrm{fg}$ & 2,15 b-e & $2,83 b-e$ & $260 \mathrm{~h}$ \\
\hline $433 \mathrm{~L}$ & $2,55 \mathrm{~cd}$ & $3,59 a b$ & $550 a-d$ & $2,05 \mathrm{c}-\mathrm{e}$ & $2,83 b-e$ & 510 a-e \\
\hline $481 P$ & $2,43 \mathrm{de}$ & $2,88 d-f$ & $440 b-f$ & 1,85 ef & $2,40 \mathrm{f}-\mathrm{h}$ & $270 \mathrm{~h}$ \\
\hline $479 P$ & $2,39 d-f$ & $2,66 \mathrm{~d}-\mathrm{g}$ & 500 b-e & $2,13 b-e$ & $2,76 \mathrm{c}-\mathrm{f}$ & $440 \mathrm{c}-\mathrm{g}$ \\
\hline $487 \mathrm{P}$ & $2,23 \mathrm{~d}-\mathrm{g}$ & $2,93 \mathrm{de}$ & 350 e-g & $2,15 b-e$ & $2,70 \mathrm{~d}-\mathrm{f}$ & 390 d-h \\
\hline $480 \mathrm{P}$ & $2,15 \mathrm{~d}-\mathrm{h}$ & $2,90 \mathrm{~d}-\mathrm{f}$ & $590 a b$ & 2,03 c-e & 2,48 e-h & 530 a-d \\
\hline $482 \mathrm{P}$ & 2,10 e-h & $2,90 \mathrm{~d}-\mathrm{f}$ & $600 a b$ & $1,90 \mathrm{~d}-\mathrm{f}$ & $2,90 \mathrm{~b}-\mathrm{d}$ & $500 \mathrm{~b}-\mathrm{e}$ \\
\hline $485 \mathrm{P}$ & $2,10 e-h$ & $3,00 \mathrm{~cd}$ & $590 a b$ & 1,79 ef & $2,51 e-h$ & $500 \mathrm{~b}-\mathrm{e}$ \\
\hline $542 P$ & $1,95 \mathrm{f}-\mathrm{h}$ & $2,53 \mathrm{f}-\mathrm{h}$ & $700 a$ & $1,55 \mathrm{f}$ & $2,78 \mathrm{c}-\mathrm{f}$ & 510 a-e \\
\hline $441 P$ & $1,92 \mathrm{f}-\mathrm{h}$ & $2,60 e-g$ & $630 a b$ & 1,78 ef & $2,44 \mathrm{f}-\mathrm{h}$ & $610 a b$ \\
\hline $448 \mathrm{~L}$ & $1,85 \mathrm{gh}$ & $2,53 \mathrm{f}-\mathrm{h}$ & $630 a b$ & $1,55 \mathrm{f}$ & $2,31 \mathrm{~g}-\mathrm{i}$ & $670 a$ \\
\hline Témoins & $1,76 \mathrm{~h}$ & $2,20 \mathrm{~h}$ & 380 e-g & $1,98 \mathrm{c}-\mathrm{e}$ & $1,98 \mathrm{ij}$ & 350 e-h \\
\hline $\begin{array}{l}\text { p.p.d.s. } \\
(P \leq 0,05)\end{array}$ & 0,44 & 0,38 & 160 & 0,44 & 0,38 & 160 \\
\hline $\begin{array}{l}\text { Moyenne } \\
\text { Vaa-L }{ }^{\infty}\end{array}$ & $3,15 * *$ & $3,50 * *$ & $409 * *$ & $2,30 * *$ & $2,83 * *$ & $423 \mathrm{~ns}$ \\
\hline $\begin{array}{l}\text { Moyenne } \\
\text { Vaa-P }\end{array}$ & 2,16 & 2,80 & 550 & 1,89 & 2,62 & 468 \\
\hline
\end{tabular}

$L$ : isolée de la luzerne; $P$ : isolée de la pomme de terre.

Les moyennes suivies par les mêmes lettres ne diffèrent pas significativement selon le test de LSD protégé à $P \leq 0,05$.

Résultats des contrastes entre les souches isolées de la luzerne (Vaa-L) et les souches isolées de la pomme de terre (Vaa-P).

* $\quad$ Significatif à $P \leq 0,01$; ns : non significatif.

\section{Pouvoir pathogène des souches de Vaa chez la pomme de terre}

Aucun flétrissement des feuilles n'a été observé, ni chez les plantes du cv. Superior inoculées avec les souches Vaa-L série 1, ni chez les plantes-témoins (tableau 4). Les plantes inoculées avec les souches Vaa-P série 1 ont présenté un flétrissement marqué des feuilles avec un indice moyen de 3,58 , à l'exception de la souche Vaa-P 437, avirulente envers sa plante-hôte avec: un indice de 1,00 (plante saine). Les autres souches $V$ aa- $P$ ont entraîné un rendement moyen en tubercules significativement inférieur $(P \leq 0,05)$ à celui des plantes-témoins (tableau 4). Enfin, les rendements moyens en tubercules des 
plantes inoculées avec les souches Vaa$P(x=12,76 \mathrm{~g})$ et Vaa-L $(x=16,62 \mathrm{~g})$ étaient significativement différents $(P \leq 0,01)$ (tableau 4).

De même avec le cv. Atlantic, la verticilliose a été uniquement observée chez les plantes inoculées avec les souches Vaa-P avec un indice moyen de flétrissement des feuilles de 2,76 (tableau 4). La souche Vaa-P 437 a aussi été avirulente envers le cv. Atlantic. Aucun symptôme n'a été observé ni chez les plantes inoculées avec les souches Vaa- $L$, ni chez les plantes-témoins (tableau 4). Chez le cv. Atlantic, nous n'avons pas noté de différence de rendement moyen en tubercules entre les plantes inoculées avec les souches Vaa-L et Vaa-P.

Tableau 4. Pouvoir pathogène des souches Vaa-L et Vaa-P envers deux cultivars de pomme de terre (série 1)

\begin{tabular}{|c|c|c|c|c|}
\hline \multirow[b]{2}{*}{ Souche } & \multicolumn{2}{|c|}{ Superior } & \multicolumn{2}{|c|}{ Atlantic } \\
\hline & $\begin{array}{c}\text { Indice de } \\
\text { flétrissement } \\
\text { des feuilles } \\
(1-5)\end{array}$ & $\begin{array}{c}\text { Rendement } \\
\text { en tubercules } \\
\text { (g) }\end{array}$ & $\begin{array}{c}\text { Indice de } \\
\text { flétrissement } \\
\text { des feuilles } \\
(1-5)\end{array}$ & $\begin{array}{c}\text { Rendement } \\
\text { en tubercules } \\
\text { (g) }\end{array}$ \\
\hline $473 \mathrm{P}^{\S}$ & $4,23 \mathrm{a}^{\dagger}$ & $13,85 \mathrm{c}$ & $2,90 \mathrm{~b}$ & $11,66 \mathrm{~b}-\mathrm{d}$ \\
\hline $170 P$ & $4,20 \mathrm{a}$ & $10,84 \mathrm{c}$ & $2,90 \mathrm{~b}$ & $10,66 \mathrm{~b}-\mathrm{d}$ \\
\hline $472 P$ & $4,10 \mathrm{a}$ & $11,13 \mathrm{c}$ & 3,67 a & $9,29 \mathrm{~d}$ \\
\hline $470 P$ & $4,00 \mathrm{a}$ & $12,22 \mathrm{c}$ & $3,47 a b$ & $11,34 \mathrm{~b}-\mathrm{d}$ \\
\hline $442 \mathrm{P}$ & 3,77 a & $11,82 \mathrm{c}$ & $3,33 a b$ & $13,46 a b$ \\
\hline $471 \mathrm{P}$ & 3,77 a & $12,05 \mathrm{c}$ & $2,03 \mathrm{c}$ & $10,12 \mathrm{~cd}$ \\
\hline $437 \quad P$ & $1,00 \mathrm{~b}$ & 17,42 a & $1,00 \mathrm{~d}$ & $12,91 \mathrm{ab}$ \\
\hline $424 \mathrm{~L}$ & $1,00 \mathrm{~b}$ & 15,42 a-c & $1,00 \mathrm{~d}$ & $13,37 a b$ \\
\hline $227 \mathrm{~L}$ & $1,00 \mathrm{~b}$ & 17,92 a & $1,00 \mathrm{~d}$ & $11,20 \mathrm{~b}-\mathrm{d}$ \\
\hline $428 \mathrm{~L}$ & $1,00 \mathrm{~b}$ & $16,84 a b$ & $1,00 \mathrm{~d}$ & $11,61 \mathrm{~b}-\mathrm{d}$ \\
\hline $426 \mathrm{~L}$ & $1,00 \mathrm{~b}$ & 14,44 a-c & $1,00 \mathrm{~d}$ & $13,25 a b$ \\
\hline $451 \mathrm{~L}$ & $1,00 \mathrm{~b}$ & $16,94 \mathrm{ab}$ & $1,00 \mathrm{~d}$ & $11,23 \mathrm{~b}-\mathrm{d}$ \\
\hline $380 \mathrm{~L}$ & $1,00 \mathrm{~b}$ & $16,77 \mathrm{ab}$ & $1,00 \mathrm{~d}$ & $10,49 \mathrm{~b}-\mathrm{d}$ \\
\hline $434 \mathrm{~L}$ & $1,00 \mathrm{~b}$ & $16,92 a b$ & $1,00 \mathrm{~d}$ & $9,52 \mathrm{~d}$ \\
\hline $224 \mathrm{~L}$ & $1,00 \mathrm{~b}$ & $18,07 \mathrm{a}$ & $1,00 \mathrm{~d}$ & $15,40 \mathrm{a}$ \\
\hline $423 \mathrm{~L}$ & $1,00 \mathrm{~b}$ & 16,44 a-c & $1,00 \mathrm{~d}$ & $12,64 a-c$ \\
\hline $403 \mathrm{~L}$ & $1,00 \mathrm{~b}$ & $16,41 \mathrm{a}-\mathrm{c}$ & $1,00 \mathrm{~d}$ & $11,73 b-d$ \\
\hline Témoins & $1,00 \mathrm{~b}$ & 17,9 a & $1,00 \mathrm{~d}$ & $10,77 b-d$ \\
\hline $\begin{array}{l}\text { p.p.d.s. } \\
(P \leq 0,05)\end{array}$ & 0,76 & 2,97 & 0,76 & 2,97 \\
\hline $\begin{array}{l}\text { Moyenne } \\
\text { Vaa-L }^{\infty}\end{array}$ & $1,00 * *$ & $16,62 * *$ & $1,00 * *$ & $12,05 \mathrm{~ns}$ \\
\hline $\begin{array}{l}\text { Moyenne } \\
\text { Vaa-P }\end{array}$ & 3,58 & 12,76 & 2,76 & 11,35 \\
\hline
\end{tabular}

$\mathrm{L}$ : isolée de la luzerne; $\mathrm{P}$ : isolée de la pomme de terre.

Les moyennes suivies par les mêmes lettres ne diffèrent pas significativement selon le test de LSD protégé à $P \leq 0,05$.

Résultats des contrastes entre les souches isolées de la luzerne (Vaa-L) et les souches isolées de la pomme de terre (Vaa-P).

** Significatif à $P \leq 0,01$; ns : non significatif. 
Les plantes du cv. Superior inoculées avec les souches Vaa-P série 2 ont montré un flétrissement très marqué des feuilles avec un indice moyen de 4,35 (tableau 5). Enfin, les rendements moyens en tubercules des plantes inoculées avec les souches Vaa-P $(x=$ $25,56 \mathrm{~g})$ et Vaa-L ( $x=73,68 \mathrm{~g})$ étaient significativement différents $(P \leq 0,01)$ (tableau 5).

Toutes les souches Vaa-P ont provoqué un flétrissement des feuilles chez les plantes inoculées du cv. Atlantic (tableau 5). À l'opposé, les souches Vaa$\mathrm{L}$ n'ont pas causé la verticilliose chez ce cultivar de pomme de terre. Enfin, les rendements moyens en tubercules des plantes inoculées avec les souches Vaa$P(x=23,79 \mathrm{~g})$ et Vaa-L $(x=42,43 \mathrm{~g})$ étaient significativement différents $(P \leq 0,01)$.

\section{Croissance des souches de Vaa à différentes températures}

Après $10 \mathrm{j} \mathrm{d}^{\prime}$ incubation à 5,10 et $15^{\circ} \mathrm{C}$, la croissance radiale moyenne des souches Vaa-L était respectivement de 1,1, 6,6 et $9,6 \mathrm{~mm}$ (fig. 1). À ces mêmes températures, la croissance radiale moyenne était respectivement de 1,4 , 7,0 et $11,1 \mathrm{~mm}$ pour les souches Vaa$P$. Les contrastes orthogonaux montrent qu'à 5,10 et $15^{\circ} \mathrm{C}$, la croissance radiale des souches Vaa-P était supérieure $(P \leq$ $0,05)$ à celle des souches Vaa-L.

La température optimale de croissance était $20^{\circ} \mathrm{C}$ pour les souches Vaa-P. Pour les souches $V a a-L$, les températures optimales étaient 20,22 et $25^{\circ} \mathrm{C}$ (fig. 1). $\mathrm{A} 20^{\circ} \mathrm{C}$, les souches Vaa-P avaient un rayon supérieur $(P \leq 0,05)$ à celui des souches Vaa-L. Par ailleurs, il n'y avait pas de différence significative de croissance radiale entre ces groupes à $22^{\circ} \mathrm{C}$. A 25 et $27^{\circ} \mathrm{C}$, sur la base des contrastes orthogonaux, les souches de Vaa-L présentaient cette fois une croissance moyenne supérieure $(P \leq 0,05)$ à celle des souches Vaa-P. II n'y a pas eu de croissance à $30^{\circ} \mathrm{C}$ pour l'ensemble des souches de Vaa (fig. 1).

Cependant, toutes les souches Vaa- $\mathrm{L}$ exposées à $30^{\circ} \mathrm{C}$ ont produit du mycélium après repiquage sur du PDA et incubation à $22^{\circ} \mathrm{C}$ durant $12 \mathrm{j}$. À I'opposé, la température de $30^{\circ} \mathrm{C}$ était

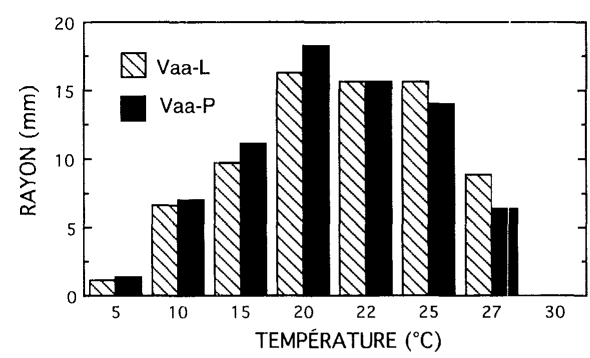

Figure 1. Effet moyen de la température sur la croissance radiale des colonies de 35 souches de Verticillium albo-atrum.

létale pour 11 des 15 souches Vaa-P; 4 souches seulement ont poussé lorsque repiquées à $22^{\circ} \mathrm{C}$.

\section{Croissance mycélienne des souches} de Vaa sur le milieu de Fries

La masse sèche du mycélium sur le milieu de Fries est généralemerit plus élevée avec les 15 souches $\mathrm{Vaa}-\mathrm{P}$ qu'avec les 20 souches Vaa-L (fig. 2). La souche Vaa-P 470 a eu la masse de mycélium lyophilisé la plus élevée (1714 mg). Cette masse était de $1020 \mathrm{mg}$ avec la souche Vaa-L 448. Les contrastes orthogonaux montrent que la masse moyenne de $825 \mathrm{mg}$ des souches Vaa-P était significativement supérieure $(P \leq 0,05)$ aux $232 \mathrm{mg}$ des souches Vaa-L.

\section{Effet du chlorate de potassium sur la croissance radiale des souches de Vaa}

En général, l'inhibition de la croissance par le chlorate de potassium était plus marquée chez les souches Vaa.P que chez les souches Vaa-L (fig. 3). Pour les souches Vaa-P, l'inhibition maximale a été de $59 \%$ (souche 472). Avec les souches Vaa-L, cette valeur était de $49 \%$ (souche 433). Par les contrastes orthogonaux, les souches Vaa-P montrent une inhibition de croissance moyenne de $40 \%$, supérieure $(P \leq 0,05)$ à celle de $20 \%$ obtenue avec les souches Vaa-L.

\section{DISCUSSION}

L'objectif de cette recherche était de démontrer la spécificité pathologique et physiologique de souches nord-américaines du $V$. albo-atrum isolées de la 
Tableau 5. Pouvoir pathogène des souches Vaa-L et Vaa-P envers deux cultivars de pomme de terre (série 2)

\begin{tabular}{|c|c|c|c|c|}
\hline \multirow[b]{2}{*}{ Souche } & \multicolumn{2}{|c|}{ Superior } & \multicolumn{2}{|c|}{ Atlantic } \\
\hline & $\begin{array}{c}\text { Indice de } \\
\text { flétrissement } \\
\text { des feuilles } \\
(1-5)\end{array}$ & $\begin{array}{l}\text { Rendement } \\
\text { en tubercules } \\
\text { (g) }\end{array}$ & $\begin{array}{c}\text { Indice de } \\
\text { flétrissement } \\
\text { des feuilles } \\
(1-5)\end{array}$ & $\begin{array}{l}\text { Rendement } \\
\text { en tubercules } \\
\text { (g) }\end{array}$ \\
\hline $441 \mathrm{P}^{\S}$ & $4,90 \mathrm{a}^{\dagger}$ & $18,32 d$ & $3,80 a b$ & $10,07 \mathrm{hi}$ \\
\hline $480 \mathrm{P}$ & $4,80 \mathrm{a}$ & $33,48 \mathrm{~d}$ & $3,20 \mathrm{~cd}$ & $36,92 \mathrm{c}-\mathrm{g}$ \\
\hline $487 \mathrm{P}$ & $4,70 a$ & $30,75 \mathrm{~d}$ & $3,60 \mathrm{bc}$ & $28,90 \mathrm{~d}-\mathrm{g}$ \\
\hline $485 \mathrm{P}$ & $4,70 a$ & $25,48 d$ & $3,40 \mathrm{bc}$ & $31,90 \mathrm{c}-\mathrm{g}$ \\
\hline $482 \mathrm{P}$ & $4,50 \mathrm{a}$ & $29,74 \mathrm{~d}$ & $3,40 \mathrm{bc}$ & $27,94 \mathrm{~d}-\mathrm{g}$ \\
\hline $542 \mathrm{P}$ & $4,40 \mathrm{a}$ & $12,39 \mathrm{e}$ & $4,20 \mathrm{a}$ & $9,69 \mathrm{i}$ \\
\hline $479 P$ & $3,70 \mathrm{~b}$ & $30,91 \mathrm{~d}$ & $2,70 \mathrm{de}$ & $23,78 \mathrm{f}-\mathrm{i}$ \\
\hline $481 P$ & $3,10 \mathrm{c}$ & $23,43 d$ & $2,30 \mathrm{e}$ & $21,15 \mathrm{~g}-\mathrm{i}$ \\
\hline $443 \mathrm{~L}$ & $1,00 \mathrm{~d}$ & $85,88 \mathrm{a}$ & $1,00 \mathrm{f}$ & $56,07 \mathrm{ab}$ \\
\hline $447 \mathrm{~L}$ & $1,00 \mathrm{~d}$ & $57,07 \mathrm{c}$ & $1,00 \mathrm{f}$ & $24,20 \mathrm{f}-\mathrm{i}$ \\
\hline $448 \mathrm{~L}$ & $1,00 \mathrm{~d}$ & $67,55 \mathrm{bc}$ & $1,00 \mathrm{f}$ & 42,77 a-e \\
\hline $228 \mathrm{~L}$ & $1,00 \mathrm{~d}$ & $77,87 a b$ & $1,00 \mathrm{f}$ & $35,04 \mathrm{c}-\mathrm{g}$ \\
\hline $435 \mathrm{~L}$ & $1,00 \mathrm{~d}$ & $75,68 a b$ & $1,00 \mathrm{f}$ & 45,20 a-e \\
\hline $445 \mathrm{~L}$ & $1,00 \mathrm{~d}$ & 73,69 a-c & $1,00 \mathrm{f}$ & $40,11 b-f$ \\
\hline $433 \mathrm{~L}$ & $1,00 \mathrm{~d}$ & $78,77 a b$ & $1,00 \mathrm{f}$ & $50,06 a b$ \\
\hline $410 \mathrm{~L}$ & $1,00 \mathrm{~d}$ & $74,97 \mathrm{ab}$ & $1,00 \mathrm{f}$ & 46,35 a-d \\
\hline $282 \mathrm{~L}$ & $1,00 \mathrm{~d}$ & 70,83 a-c & $1,00 \mathrm{f}$ & $37,21 \mathrm{c}-\mathrm{g}$ \\
\hline $450 \mathrm{~L}$ & $1,00 \mathrm{~d}$ & 74,52 a-c & $1,00 \mathrm{f}$ & 47,25 a-c \\
\hline Témoins & $1,00 \mathrm{~d}$ & $84,05 a b$ & $1,00 \mathrm{f}$ & $60,66 \mathrm{a}$ \\
\hline $\begin{array}{l}\text { p.p.d.s. } \\
(P \leq 0,05)\end{array}$ & 0,51 & 18,05 & 0,51 & 18,05 \\
\hline $\begin{array}{l}\text { Moyenne } \\
\text { Vaa- } L^{\infty}\end{array}$ & $1,00 * *$ & $73,68 * *$ & $1,00 * *$ & $42,43 * *$ \\
\hline $\begin{array}{l}\text { Moyenne } \\
\text { Vaa-P }\end{array}$ & 4,35 & 25,56 & 3,32 & 23,79 \\
\hline
\end{tabular}

$\mathrm{L}$ : isolée de la luzerne; $\mathrm{P}$ : isolée de la pomme de terre.

Les moyennes suivies par les mêmes lettres ne diffèrent pas significativement selon le test de LSD protégé à $P \leq 0,05$.

$\infty \quad$ Résultats des contrastes entre les souches isolées de la luzerne (Vaa- $L$ ) et les souches isolées de la pomme de terre (Vaa-P).

* $\quad$ Significatif à $P \leq 0,01$.

luzerne et de la pomme de terre. Par ces résultats, deux groupes de souches de Vaa se distinguent sur la base de leur pouvoir pathogène et de leur croissance radiale dans des conditions de milieu et de température définies. D'une part, lorsque inoculées chez la luzerne, les souches Vaa-L sont plus virulentes que les souches Vaa-P. D'autre part, seules les souches Vaa-P ont entraîné le flétrissement de la pomme de terre. Aucune souche Vaa- $L$ n'a produit de symptômes de verticilliose chez la pomme de terre, pas même chez le cv. Superior pourtant très sensible à la verticilliose selon Platt (1986).

Que ce soit chez la luzerne ou chez la pomme de terre, I'indice de flétrissement des feuilles a permis de distin- 


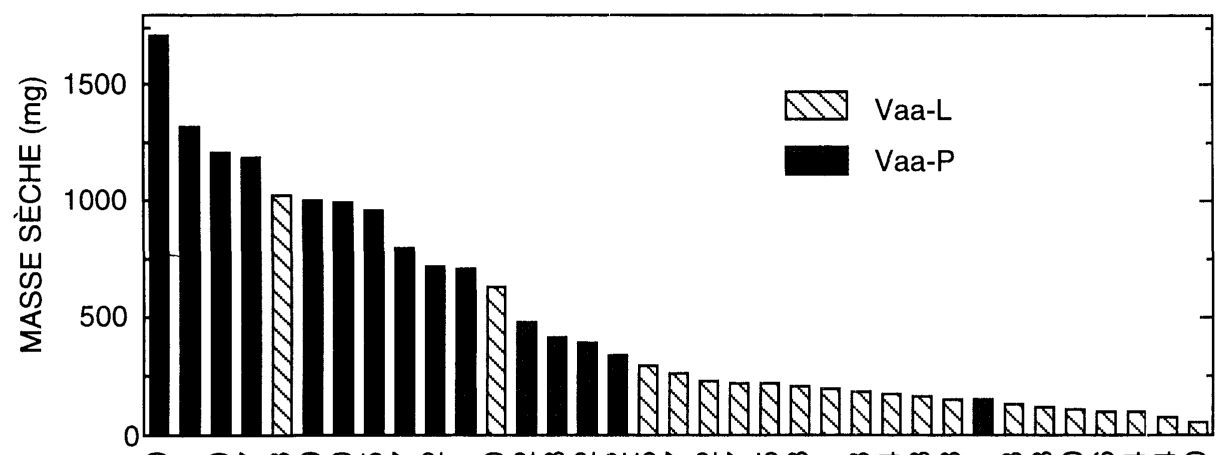

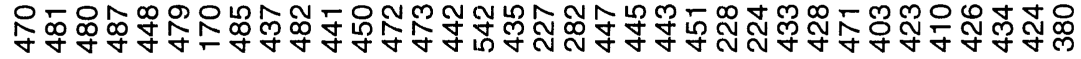

SOUCHE

Figure 2. Réponse de la croissance du mycélium de 35 souches de Verticillium albo-atrum inoculées sur le milieu de Fries.

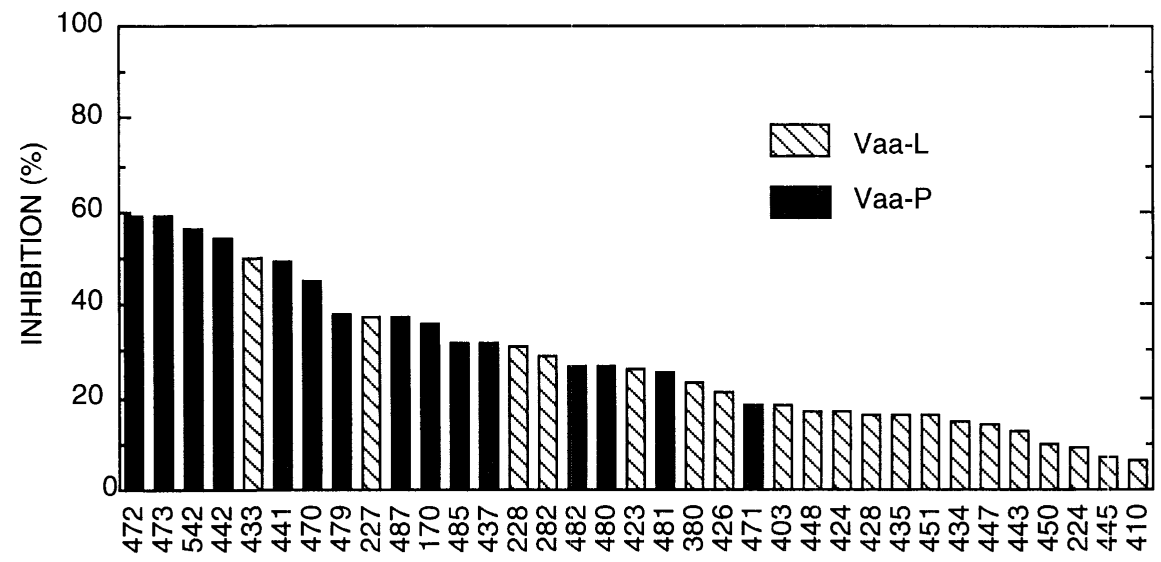

SOUCHE

Figure 3. Effet du chlorate de potassium sur l'inhibition de la croissance radiale de 35 souches de Verticillium albo-atrum inoculées dans le milieu de Puhalla.

guer les groupes Vaa-L et Vaa-P. Ces résultats corroborent ceux de Correll et al. (1988) à l'effet que la verticilliose est plus grave chez la luzerne inoculée avec des souches de Vaa-L qu'avec d'autres souches. Par ailleurs, nos résultats se distinguent de ceux de quelques études. Ainsi, Heale et Isaac (1963) ont rapporté que leurs souches $V a a-P$ ne pouvaient pas causer la verticilliose chez la luzerne. Or, dans nos travaux, quelques souches issues de la pomme de terre ont provoqué un flétrissement des feuilles et un brunissement des racines significatifs chez la luzerne cv. Saranac (sensible) par rapport aux plantestémoins. Autre divergence, Isaac et Lloyd (1959) ont obtenu $88 \%$ de plantes infectées lorsque la pomme de terre est inoculée avec des souches Vaa-L. Dans nos expériences, toutes les souches Vaa-L ont été avirulentes chez les deux cultivars de pomme de terre. A cet égard, nos résultats corroborent les observations de Christen et French (1982) qui n'ont pas pu reproduire la verticilliose chez la pomme de terre inoculée avec des souches Vaa-L. 
Avec la luzerne, la biomasse sèche aérienne $\mathrm{n}^{\prime}$ a pas permis la discrimination des souches Vaa- $L$ et Vaa-P, en particulier avec le cV. Oneida VR (résistant). Chez le cv. Saranac (sensible), seulement 6 souches sur 10 de Vaa-L de la série 1 ont réduit significativement le rendement par rapport aux plantestémoins non inoculées. Dans la série 2 (tableau 3), 2 souches sur 10 de Vaa-L et 5 souches sur 8 de Vaa-P inoculées chez le cv. Saranac ont entraîné un rendement supérieur $(P \leq 0,05)$ aux plantes-témoins.

À l'opposé, chez la pomme de terre cv. Superior, le rendement en tubercules est discriminant. Toutes les souches Vaa- $P$ virulentes réduisent $(P \leq 0,05)$ le rendement par rapport au témoin et par rapport à toutes les souches $V a a-L$ de la série 2 (tableau 5). Par contre, chez le cv. Atlantic (résistant), surtout dans la série 1 , le rendement ne permet de distinguer ni les souches Vaa- $L$, ni les souches $V a a-P$ des plantes-témoins non inoculées.

Chez la pomme de terre, avec les souches de la série 1 inoculées en été, les températures diurnes de la serre de $24-30^{\circ} \mathrm{C}$ ont réduit les rendements dans tous les traitements (maximum de $\left.18,07 \mathrm{~g} \mathrm{pot}^{-1}\right)$. Cette température a probablement masqué l'effet des souches Vaa-P chez le cv. Atlantic. Par contre, chez les plantes inoculées avec les souches de la série 2 en hiver où les températures diurnes étaient de $18-22^{\circ} \mathrm{C}$, le rendement maximum en tubercules était de $85,88 \mathrm{~g} \mathrm{pot}^{-1}$. Le rendement moyen chez les plantes inoculées avec les souches Vaa-P était plus faible qu'avec les souches $V a a-L$ ou des plantes-témoins. Dans la série 2, la baisse du rendement par rapport aux plantes-témoins chez le cultivar Superior atteignait $70 \%$ avec les souches Vaa$P$, et seulement $12 \%$ avec les souches Vaa-L.

Chez la luzerne, la souche Vaa-L 448, virulente dans les travaux de Correll et al. (1988), était pratiquement avirulente chez les cv. Saranac et Oneida VR dans nos expériences. Aussi, la souche VaaP 437, obtenue de l'île-du-PrinceÉdouard était totalement avirulente envers la pomme de terre.
La température optimale de croissance des souches Vaa-P est de $20^{\circ} \mathrm{C}$ sur du PDA, alors que cet optimum est partagé entre 20,22 et $25^{\circ} \mathrm{C}$ pour les souches Vaa-L. Cette gamme de températures optimales des souches $\mathrm{Vaa}-\mathrm{L}$ rejoint les observations de Christen et French (1982). Sur du PDA, aucune des souche Vaa n'a poussé à $30^{\circ} \mathrm{C}$. À I'opposé des souches Vaa- $P$, toutes les souches $V a a-L$ ont repris leur croissance lorsque répiquées à $22^{\circ} \mathrm{C}$. Ce résultat corrobore les observations de Gondran (1984) qui rapporte une croissance des souches Vaa- $L$ ensemencées sur une gélose à la pomme de terre et au saccharose et incubées à $30^{\circ} \mathrm{C}$. Par ailleurs, Christen et Peaden (1981) ont isolé, d'une luzerne infectée par le Vaa, l'agent pathogène dans certains échantillons incubés à $33^{\circ} \mathrm{C}$ et non à $35^{\circ} \mathrm{C}$. Ainsi, la température de $30^{\circ} \mathrm{C}$ n'est pas létale pour les souches $\mathrm{Vaa}-\mathrm{L}$, mais le serait pour la plupart des souches Vaa-P.

La culture des souches Vaa-L et Vaa$P$ sur le milieu de Fries et sur le milieu à base de chlorate de potassium a donné des résultats opposés. D'une part, sur le milieu de Fries, la masse moyenne du mycélium lyophilisé des souches Vaa-P (825 mg) est nettement supérieure à la moyenne des souches Vaa-L $(232$ $\mathrm{mg}$ ). Nous supposons que, dans ce milieu, les souches Vaa- $L$ ont sécrété des métabolites auto-inhibiteurs. D'autre part, l'inhibition de la croissance sur le milieu amendé au chlorate de potassium a été significativement plus marquée avec les souches $V a a-P$ qu'avec les souches Vaa-L.

Dans notre étude, la moins grande inhibition de croissance de la plupart des souches Vaa-L pourrait s'expliquer par l'inhibition de la biosynthèse de la nitrate réductase ou par son mauvais fonctionnement. Cette enzyme réduit le chlorate de potassium en chlorite de potassium, un produit toxique pour les champignons (Cove 1976). Néanmoins, la réduction du chlorate de potassium en chlorite de potassium ne serait pas suffisante pour qu'un champignon soit tué, comme en fait foi le cas de l'Aspergillus nidulans (Cove 1976). Quoique le chlorate de potassium puisse produire des mutations chez le Vaa (Correll et al. 
1988), aucun changement morphologique ne s'est produit avec nos souches. Cependant, les souches Vaa-P ont présenté un niveau moyen d'inhibition plus accentué, soit $40 \%$ contre $20 \%$ chez les souches Vaa-L.

Cette étude met en évidence que les souches Vaa- $L$ et Vaa- $P$, agents pathogènes de la verticilliose de la luzerne et de la pomme de terre, forment deux groupes distincts par leur virulence. Le groupe luzerne est spécifique à cette plante alors que le groupe pomme de terre est plus virulent chez la pomme de terre que chez la luzerne. Sur du PDA, la plupart des souches Vaa-P sont tuées à $30^{\circ} \mathrm{C}$. De plus, avec le milieu de Fries, nous avons obtenu une différence nette de croissance entre les souches Vaa-L et les souches Vaa-P. Ces différences significatives entre les souches $V a a-L$ et Vaa-P sont aussi corroborées par les résultats obtenus au niveau génétique par la technique d'ADN polymorphe amplifié au hasard (RAPD) (Barasubiye et al., résultats non publiés).

\section{REMERCIEMENTS}

Les auteurs remercient $M$. Jean-Guy Martin pour sa participation à l'exécution des travaux, ainsi que M. Gilbert Banville qui nous a fourni la semence de pomme de terre. Nous tenons aussi à remercier tous les chercheurs qui nous ont fourni des souches de V. albo-atrum et le Programme Canadien des bourses de la Francophonie qui a accordé une bourse à $T$. Barasubiye.

\section{RÉFÉRENCES}

Aubé, C. et W.E. Sackston. 1964. Verticillium wilt of forage legumes in Canada. Can. J. Plant Sci. 44: 427-432.

Ayers, G.W. et R.R. Hurst. 1939. Verticillium wilt of potatoes in Prince Edward Island. Sci. Agric. 19: 722-735.

Berkeley, G.H., G.O. Madden et R.S. Willison. 1931. Verticillium wilts in Ontario. Sci. Agric. 11: 739-759.

Christen, A.A. et R.C. French. 1982. Growth and pathogenicity of alfalfa strain of Verticillium albo-atrum. Plant Dis. 66: 416418.
Christen, A.A. et R.N. Peaden. 1981 "Verticillium wilt in alfalfa. Plant Dis. 65: 319321.

Christen, A.A., R.N. Peaden, G.P. Harris et J.B. Heale. 1983. Virulence of North American and European isolates of Verticillium albo-atrum on alfalfa cultivars. Phytopathology 73: 1051-1054.

Correll, J.C., T.R. Gordon et A.H. McCain. 1988. Vegetative compatibility and pathogenicity of Verticillium albo-atrum. Phytopathology 78: 1017-1021.

Corsini, D.L., J.R. Davis et J.J. Pavek. 1985. Stability of resistance of potato to strains of Verticillium dahliae from different vegetative compatibility groups. Plant Dis. 69: 980-982.

Cove, D.J. 1976. Chlorate toxicity in Aspergillus nidulans studies of mutants altered in nitrate assimilation. Mol. Gen. Genet. 146: 147-159.

Dhingra, O.D. et J.B. Sinclair. 1985. Basic plant pathology methods. CRC Press Inc., Boca Raton, Floride. 355 pp.

Flood, J., I. Isaac et J.M. Milton. 1978. Reactions of some cultivars of lucerne to various isolates of Verticillium albo-atrum. Plant Pathol. 27: 166-169.

Gagné, S. et C. Richard. 1982. La verticilliose de la luzerne en Amérique du Nord. Can. J. Plant Pathol. 4: 47-53.

Gondran, J. 1984. La verticilliose de la luzerne : Détermination de l'agent causal, biologie du parasite, répartition géographique, dégâts et méthodes de lutte. Thèse de Docteur-ingénieur. Université de Poitiers, France. 156 pp.

Gordon, T.R., J.C. Correll, D.G. Gilchrist et A.N. Martensen. 1989. Verticillium wilt of alfalfa in California. Plant Dis. 73: 18-20.

Gossen, B.D. et G.D. Jesperson. 1990. Verticillium wilt of irrigated alfalfa in Saskatchewan, 1987-89. Can. Plant Dis. Surv. 70: 129-131.

Graham, J.H., R.N. Peaden et D.W. Evans. 1977. Verticillium wilt of alfalfa found in the United States. Plant Dis. Rep. 61: 337340.

Heale, J.B. et I. Isaac. 1963. Wilt of lucerne caused by species of Verticillium. IV. Pathogenicity of $V$. albo-atrum and $V$. dahliae to lucerne and other crops; spread and survival of $V$. albo-atrum in soil and in weeds; effect upon lucerne production. Ann. Appl. Biol. 52: 439-451.

Isaac, I. et A.T.E. Lloyd. 1959. Wilt of lucerne caused by species of Verticillium. II. Seasonal cycle disease; range of pathogenicity; host-parasite relations; effects of seed dressings. Ann. Appl. Biol. 47: 673-684. 
Kreitlow, K.W. 1962. Verticillium wilt of alfalfa. A destructive disease of Britain and Europe not yet observed in the United States. U.S. Dep. Agric. Agric. Res. Serv. ARS 34-20. 15 pp.

Martin, R.A. et P. Boswall. 1989. First occurrence report of Verticillium wilt of alfalfa on Prince Edward Island. Can. Plant Dis. Surv. 69: 51.

McKeen, C.D. et H.J. Thorpe. 1981. Verticillium wilt of potato in southwestern Ontario and survival of Verticillium alboatrum and $V$. dahliae in field soil. Can. J. Plant Pathol. 3: 40-46.

Nachmias, A. et J. Krikun. 1985. Verticillium wilt of potato in Israel. Am. Potato J. 62: 201-205.

Nicholls, H., C. Richard et J.-G. Martin. 1987. Verticillium wilt of alfalfa in Quebec. Can. Plant Dis. Surv. 67: 17-21.
Platt, H.W. 1986. Varietal response and crop loss due to Verticillium wilt of potato caused by $V$. albo-atrum. Phytoprotection 67: 123-127.

Platt, H.W. et J.B. Sanderson. 1987. Comparison of inoculation methods for field studies on varietal response to verticillium wilt of potatoes. Am. Potato J. 64: 87-92.

Puhalla, J.E. 1985. Classification of strains of Fusarium oxysporum on the basis of vegetative compatibility. Can. J. Bot. 63: 179-183.

SAS Institute Inc. 1990. SAS/STAT User's guide: version $6.0,4^{\text {th }} \mathrm{Ed}$. SAS Institute Inc. Cary, Caroline du Nord. 943 pp.

Steel, R.G.D. et J.M. Torrie. 1980. Principles and procedures of statistics : a biometrical approach. $2^{\mathrm{e}}$ édition, McGraw Hill Book Co., New York. 633 pp. 